\title{
Two Mode Resonator and Contact Model for Standing Wave Piezomotor
}

\author{
Andersen, Brian; Blanke, Mogens; Helbo, J.
}

Published in:

Proceedings of the $18<$ sup $>$ th $<$ sup $>$ ASME Biennial Conference on Mechanical Vibration and Noise

Publication date:

2001

Document Version

Peer reviewed version

Link back to DTU Orbit

Citation (APA):

Andersen, B., Blanke, M., \& Helbo, J.t(2001). Two Mode Resonator and Contact Model for Standing Wave Piezomotor. In Proceedings of the 18 ASME Biennial Conference on Mechanical Vibration and Noise American Society of Mechanical Engineers.

\section{General rights}

Copyright and moral rights for the publications made accessible in the public portal are retained by the authors and/or other copyright owners and it is a condition of accessing publications that users recognise and abide by the legal requirements associated with these rights.

- Users may download and print one copy of any publication from the public portal for the purpose of private study or research.

- You may not further distribute the material or use it for any profit-making activity or commercial gain

- You may freely distribute the URL identifying the publication in the public portal

If you believe that this document breaches copyright please contact us providing details, and we will remove access to the work immediately and investigate your claim. 


\section{TWO-MODE RESONATOR AND CONTACT MODEL FOR STANDING WAVE PIEZOMOTOR}

\author{
Brian Andersen \\ Department of Control Engineering \\ Aalborg University \\ Fredrik Bajers Vej 7C \\ DK-9220 Aalborg \\ Denmark \\ Email: ba@control.auc.dk \\ TIf: +4596358756
}

\author{
Mogens Blanke \\ Department of Automation \\ Technical University of Denmark \\ Building 326/327 \\ DK-2800 Kongens Lyngby \\ Denmark \\ Email: blanke@iau.dtu.dk
}

\author{
Jan Helbo \\ Department of Control Engineering \\ Aalborg University \\ Fredrik Bajers Vej 7C \\ DK-9220 Aalborg \\ Denmark \\ Email: helbo@control.auc.dk
}

\begin{abstract}
The paper presents a model for a standing wave piezoelectric motor with a two bending mode resonator. The resonator is modelled using Hamilton's principle and the Rayleigh-Ritz method. The contact is modelled using the Lagrange Multiplier method under the assumption of slip and it is showed how to solve the set of differential-algebraic equations. Detailed simulations show resonance frequencies as function of the piezoelement's position, tip trajectories and contact forces. The paper demonstrates that contact stiffness and stick should be included in such model to obtain physically realistic results and a method to include stick is suggested.
\end{abstract}

\section{INTRODUCTION}

Most two mode standing wave piezoelectric motors excites a longitudinal mode and a bending mode to generate elliptical trajectories. This paper presents a method where both first and second bending modes are excited. A sketch of the motor which is currently being constructed is shown in Figure 1. The principle used in this motor is taken from the design of a spherical piezoelectric motor (Andersen et al. 2000). To predict the performance of a piezomotor an important issue is the contact model. The contact problem for travelling wave motors has been analysed by many authors (Wallaschek 1998) whereas the contact for the intermittent contact type motor has been analysed in smaller extent. Zharii (1996) proposed a method based on Hertz theory and Fung et al. (1999) solved the contact problem using the Lagrange Multiplier method. Gear et al. (1985) proposed a method for a numerical solution to this problem, Hatman et al. (1996) extended the method to include friction at the contact interface.

In this paper the resonator is modelled using Hamilton's principle together with a Rayleigh-Ritz representation of the assumed modes. The effect of the piezoelement's position on the tip trajectories and resonance frequencies are studied. The contact constraint is modelled using the Lagrange multiplier method under the assumption of slip. Using earlier methods (Hatman et al. 1996) and (Fung et al. 1999) to solve the system equations, simulation results are presented. The quality of the results are discussed, and it is shown that the contact force can hardly be correct. The indication is that the commonly accepted way of using the Lagrange Multiplier method falls short on this problem. The remedial is inclusion of contact stiffness in the model. Also stick is included to improve the slip-stick model. The paper presents these enhancements of a new piezomotor model.

\section{DERIVATION OF BENDING VIBRATIONS FOR THE RESONATOR}

Figure 1 shows the piezoresonator with piezoelements attached to the beam. The poling direction of the piezoceramic is indicated by the arrow $\mathrm{P}$ and causes the upper piezoelements to expand when the applied voltages are positive relative to ground whereas the lower elements will contract for the same voltage. When the applied voltage becomes negative relative to ground the situation is reversed. The voltages $U_{A}$ and $U_{B}$ are formed by 


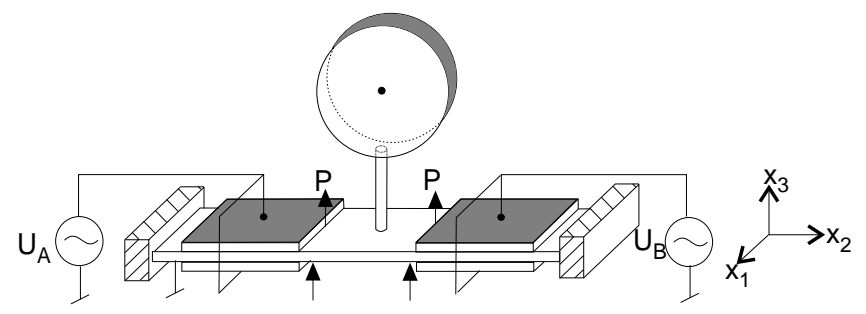

Figure 1. PIEZORESONATOR CLAMPED AT BOTH ENDS.

superposing two sinusoidal voltage signals

$$
\begin{aligned}
& U_{A}=U_{1} \sin \left(\omega_{1} t+\phi\right)+U_{2} \sin \left(\omega_{2} t\right) \\
& U_{B}=U_{1} \sin \left(\omega_{1} t+\phi\right)-U_{2} \sin \left(\omega_{2} t\right)
\end{aligned}
$$

where $U_{1}$ and $U_{2}$ are amplitudes and $\phi$ is an adjustable phase. Setting $U_{2}=0$ causes excitation of first bending mode and setting $U_{1}=0$ causes excitation of second bending mode. If both $U_{1} \neq 0$, $U_{2} \neq 0$ and $\frac{\omega_{2}}{\omega_{1}}=2$ the trajectory of the tip will look like a number 8.

The derivation of the resonator model takes a starting point in Hamilton's principle for an electromechanical system (Hagood et al. 1990)

$$
\int_{t_{1}}^{t_{2}}\left[\delta\left(T-U+W_{e}\right)+\delta W\right] d t=0
$$

where $\delta$ is the variational operator, $T$ is kinetic energy, $U$ is strain energy, $W_{e}$ is electric energy and $W$ represents energy from applied external point forces, torques and applied charges. Expressions for the energy terms in (3) are given as follows.

$$
\begin{aligned}
& T=\int_{V_{s}} \frac{1}{2} \rho_{s} \dot{\mathbf{u}}^{T} \dot{\mathbf{u}} d v+\int_{V_{p}} \frac{1}{2} \rho_{p} \dot{\mathbf{u}}^{T} \dot{\mathbf{u}} d v+T_{t i p} \\
& U=\int_{V_{s}} \frac{1}{2} \mathbf{S}^{T} \mathbf{T} d v+\int_{V_{p}} \frac{1}{2} \mathbf{S}^{T} \mathbf{T} d v \\
& W_{e}=\int_{V_{p}} \frac{1}{2} \mathbf{E}^{T} \mathbf{D} d v \\
& \delta W=\sum_{j=1}^{n_{f}} \delta \mathbf{u}\left(\mathbf{x}_{j}\right) \cdot \mathbf{f}\left(\mathbf{x}_{j}\right)+\sum_{j=1}^{n_{\tau}} \delta \theta\left(\mathbf{x}_{j}\right) \tau\left(\mathbf{x}_{j}\right)-\sum_{j=1}^{2} \delta \varphi_{j} q_{j}
\end{aligned}
$$

where $\mathbf{u}$ is the displacement vector, $\mathbf{S}$ and $\mathbf{T}$ are vectors of strain and stress, $\mathbf{E}$ and $\mathbf{D}$ denotes electric field and electric displacement respectively, $\mathbf{f}\left(\mathbf{x}_{j}\right)$ is a force applied at a point, $\tau$ is a torque resulting in a rotation $\theta$ and $\varphi_{j}$ and $q_{j}$ are electric potential and charge applied to the piezoceramic set $j$. The kinetic energy for the tip equals

$$
T_{\text {tip }}=\frac{1}{2} I_{t i p} \dot{\theta}^{2}+\frac{1}{2} M_{t i p} \dot{u}_{3}^{2}(L / 2)
$$

where $I_{t i p}$ is the tip's moment of inertia about it's end and $\dot{u}_{3}(L / 2)$ is the velocity in $x_{3}$ direction at the centre of the beam. The constitutive relations for the beam and the piezoceramic are given by

$$
\begin{gathered}
\mathbf{T}=\mathbf{c}_{S} \mathbf{S} \\
{\left[\begin{array}{l}
\mathbf{D} \\
\mathbf{T}
\end{array}\right]=\left[\begin{array}{cc}
\varepsilon^{S} & \mathbf{e} \\
-\mathbf{e}^{T} & \mathbf{c}^{E}
\end{array}\right]\left[\begin{array}{l}
\mathbf{E} \\
\mathbf{S}
\end{array}\right]}
\end{gathered}
$$

The strain-displacement and field-potential relationship are given by

$$
\begin{array}{r}
\mathbf{S}=\mathbf{L}_{u} \mathbf{u}\left(x_{2}, x_{3}\right) \\
\mathbf{E}=\mathbf{L}_{\varphi} \varphi\left(x_{2}, x_{3}\right)
\end{array}
$$

where $\mathbf{L}_{u}$ and $\mathbf{L}_{\varphi}$ are operators for the elasticity problem and the electric field. Here the beam is modelled as a BernoulliEuler beam. To increase accuracy of the strain model within the piezoelements the stress free condition through the thickness of beam and piezoelements is modelled by including the strain component $\mathrm{i}$ the thickness direction. Then the operator for the elasticity becomes (Hagood et al. 1990)

$$
\mathbf{L}_{u}=\left[\begin{array}{ccc}
0 & 0 & 0 \\
0 & 0 & -x_{3} \frac{\partial^{2}}{\partial x_{2}^{2}} \\
0 & 0 & v x_{3} \frac{\partial^{2}}{\partial x_{2}^{2}} \\
0 & 0 & 0 \\
0 & 0 & 0 \\
0 & 0 & 0
\end{array}\right]
$$

where $v$ is Possion's ratio. The relation between electric potential and electric field is given as $\mathbf{E}=-\nabla \varphi$. Here we assume the potential is constant in the $x_{1}$ and $x_{2}$ directions, therefore the operator for the electric field can be formulated as

$$
\mathbf{L}_{\varphi}=\left[\begin{array}{c}
0 \\
0 \\
-\frac{\partial}{\partial x_{3}}
\end{array}\right]
$$

Now the Rayleigh-Ritz formulation is used to express the displacement $\mathbf{u}\left(x_{2}, x_{3}\right)$ as a superposition of vibration modes as fol- 


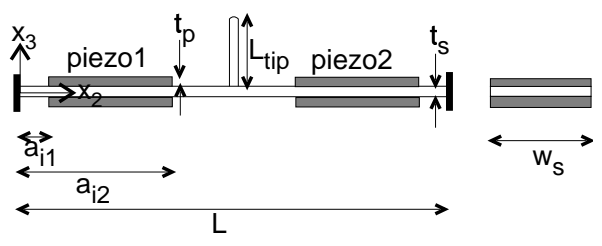

Figure 2. GEOMETRY OF THE PIEZORESONATOR. THE INDEX i REFERS TO SET 1 OR 2 OF THE PIEZOELEMENTS.

lows

$$
\mathbf{u}=\left[\begin{array}{c}
0 \\
u_{2} \\
u_{3}
\end{array}\right]=\Psi_{r} \mathbf{r}=\left[\begin{array}{ccc}
0 & \ldots & 0 \\
-x_{3} \frac{\partial u_{3_{1}}\left(x_{2}\right)}{\partial x_{2}} & \ldots & -x_{3} \frac{\partial u_{3_{n}}\left(x_{2}\right)}{\partial x_{2}} \\
u_{3_{1}}\left(x_{2}\right) & \ldots & u_{3_{n}}\left(x_{2}\right)
\end{array}\right]\left[\begin{array}{c}
r_{1}(t) \\
\vdots \\
r_{n}(t)
\end{array}\right]
$$

where $u_{2}$ is the displacement due to rotation of cross sections of the beam and $\mathbf{r}$ is the vector of generalized displacement coordinates. The mode shapes used here are the eigenmodes for a clamped-clamped Bernoulli-Euler beam. The electric potential in the piezoceramic is assumed to decrease linearly through the thickness of the elements therefore only one mode shape for each set of piezoelements is necessary to describe the potential

$$
\varphi\left(x_{2}, x_{3}, t\right)=\boldsymbol{\Psi}_{v} \mathbf{v}=\left[\varphi_{1}\left(x_{2}, x_{3}\right) \varphi_{2}\left(x_{2}, x_{3}\right)\right]\left[\begin{array}{l}
v_{1}(t) \\
v_{2}(t)
\end{array}\right]
$$

where $\mathbf{v}$ is the vector of generalized electrical coordinates. In this case the generalized electrical coordinates $v_{1}(t)$ and $v_{2}(t)$ are equal to the applied input voltages $U_{A}(t)$ and $U_{B}(t)$. This is seen from the following potential distributions which are used

$$
\varphi_{i}\left(x_{2}, x_{3}\right)= \begin{cases}0 & x_{2}<a_{i 1} \\ \frac{x_{3}-\frac{t_{s}}{2}}{t_{p}} & a_{i 1}<x_{2}<a_{i 2}, x_{3}>0 \\ \frac{-x_{3}-\frac{t_{s}}{2}}{t_{p}} & a_{i 1}<x_{2}<a_{i 2}, x_{3}<0 \\ 0 & x_{2}>a_{i 2}\end{cases}
$$

where $a_{i j}$ is defined on Figure 2. Thus, substituting $x_{3}=\frac{t_{s}}{2}+t_{p}$, corresponding to the surface of the upper piezoelement, results in $\varphi_{1}=1 \cdot v_{1}(t)=U_{A}(t)$ for the set of piezoelements to the left.

Now the equations for the resonator can be derived; starting with equation (3) substituting the energy expressions equation (4)-(7), using the constitutive equations (9)-(10) and calculating the variations gives

$$
\begin{aligned}
& \int_{t_{1}}^{t_{2}}\left(\int_{V_{s}}-\rho_{s} \delta \mathbf{u}^{T} \ddot{\mathbf{u}} d v+\int_{V_{p}}-\rho_{p} \delta \mathbf{u}^{T} \ddot{\mathbf{u}} d v-I_{t i p} \delta \theta \ddot{\theta}-M_{t i p} \delta u_{3} \ddot{u}_{3}(L / 2)-\right. \\
& \int_{V_{s}} \delta \mathbf{S}^{T} \mathbf{c}_{s} \mathbf{S} d v+\int_{V_{p}} \delta \mathbf{S}^{T} \mathbf{e}^{T} \mathbf{E} d v-\int_{V_{p}} \delta \mathbf{S}^{T} \mathbf{c}^{E} \mathbf{S} d v+\int_{V_{p}} \delta \mathbf{E}^{T} \varepsilon^{S} \mathbf{E} d v+ \\
& \left.\int_{V_{p}} \delta \mathbf{E}^{T} \mathbf{e} \mathbf{S} d v+\sum_{j=1}^{n_{f}} \delta u_{3}\left(\mathbf{x}_{j}\right) \cdot f_{3}\left(\mathbf{x}_{j}\right)+\sum_{j=1}^{n_{\tau}} \delta \theta\left(\mathbf{x}_{j}\right) \tau\left(\mathbf{x}_{j}\right)-\sum_{j=1}^{2} \delta \varphi_{j} q_{j}\right) d t=0
\end{aligned}
$$

where the first 4 terms are variations of kinetic energy, the next 3 terms are variations of strain energy, followed by 2 terms for variations of electric energy and the last 3 terms are identical to equation (7) except that only the $x_{3}$ component of forces is included. To proceed the calculation further the strain-displacement and field-potential relations in equations (11) and (12) together with the representations of displacement and potential in equations (15) and (16) are inserted in the above equation

$$
\begin{aligned}
& \int_{t_{1}}^{t_{2}}\left(\delta \mathbf { r } ^ { T } \left[\int_{V_{s}}-\rho_{s} \boldsymbol{\Psi}_{r}^{T} \boldsymbol{\Psi}_{r} \ddot{\mathbf{r}}+\int_{V_{p}}-\rho_{p} \boldsymbol{\Psi}_{r}^{T} \boldsymbol{\Psi}_{r} \ddot{\mathbf{r}}-I_{t i p} \frac{\partial \Psi_{r 3}^{T}}{\partial x_{2}} \frac{\partial \Psi_{r 3}}{\partial x_{2}} \ddot{\mathbf{r}}-\right.\right. \\
& M_{t i p} \boldsymbol{\Psi}_{r 3}^{T} \boldsymbol{\Psi}_{r 3} \ddot{\mathbf{r}}-\int_{V_{s}}\left(\mathbf{L}_{u} \boldsymbol{\Psi}_{r}\right)^{T} \mathbf{c}_{s} \mathbf{L}_{u} \boldsymbol{\Psi}_{r} \mathbf{r}+\int_{V_{p}}\left(\mathbf{L}_{u} \boldsymbol{\Psi}_{r}\right)^{T} \mathbf{e}^{T} \mathbf{L}_{\varphi} \boldsymbol{\Psi}_{v} \mathbf{v}- \\
& \left.\int_{V_{p}}\left(\mathbf{L}_{u} \boldsymbol{\Psi}_{r}\right)^{T} \mathbf{c}^{E} \mathbf{L}_{u} \boldsymbol{\Psi}_{r} \mathbf{r}+\sum_{j=1}^{n_{f}} \boldsymbol{\Psi}_{r 3}^{T} f_{3}\left(\mathbf{x}_{j}\right)+\sum_{j=1}^{n_{t}} \frac{\partial \boldsymbol{\Psi}_{r 3}}{\partial x_{2}} \tau_{1}\left(\mathbf{x}_{j}\right)\right]+ \\
& \left.\delta \mathbf{v}^{T}\left[\int_{V_{p}}\left(\mathbf{L}_{\varphi} \boldsymbol{\Psi}_{v}\right)^{T} \mathcal{\varepsilon}^{S} \mathbf{L}_{\varphi} \boldsymbol{\Psi}_{v} \mathbf{v}+\int_{V_{p}}\left(\mathbf{L}_{\varphi} \boldsymbol{\Psi}_{v}\right)^{T} \mathbf{e} \mathbf{L}_{u} \boldsymbol{\Psi}_{r} \mathbf{r}-\sum_{j=1}^{2} \boldsymbol{\Psi}_{v}^{T} q_{j}\right]\right) d t=0
\end{aligned}
$$

where $\boldsymbol{\Psi}_{r 3}$ is the third row of $\boldsymbol{\Psi}_{r}$ and $\theta$ is approximated with the slope $\frac{\partial \Psi_{r 3}}{\partial x_{2}} \mathbf{r}$ and $d v$ has been omitted for convenience. Since arbitrary variations are allowed to both $\delta \mathbf{r}$ and $\delta \mathbf{v}$ both the first and second bracket must equal zero. This gives two equations where the coefficients are constant in time

$$
\begin{array}{ll}
\mathbf{M} \ddot{\mathbf{r}}+\mathbf{K r}-\boldsymbol{\Gamma v}=\sum_{j=1}^{n_{f}} \mathbf{Q}_{F}\left(\mathbf{x}_{j}\right)+\sum_{j=1}^{n_{t}} \mathbf{Q}_{T}\left(\mathbf{x}_{j}\right) & \text { Actuator Eq. } \\
\mathbf{C}_{p} \mathbf{v}+\boldsymbol{\Gamma}^{T} \mathbf{r}=\sum_{j=1}^{2} \mathbf{Q}_{j} & \text { Sensor Eq. }
\end{array}
$$

with

$$
\begin{array}{ll}
\mathbf{M}=\mathbf{M}_{s}+\mathbf{M}_{p}+\mathbf{M}_{t i p} & \text { Total mass } \\
\mathbf{K}=\mathbf{K}_{s}+\mathbf{K}_{p} & \text { Total stiffness } \\
\mathbf{M}_{t i p}=-I_{t i p} \frac{\partial \boldsymbol{\Psi}_{r 3}^{T}}{\partial x_{2}} \frac{\partial \boldsymbol{\Psi}_{r 3}}{\partial x_{2}}-M_{t i p} \boldsymbol{\Psi}_{r 3}^{T} \boldsymbol{\Psi}_{r 3} & \text { Tip equivalent mass }
\end{array}
$$

$$
\mathbf{Q}_{F}\left(\mathbf{x}_{j}\right)=\boldsymbol{\Psi}_{r 3}^{T}\left(\mathbf{x}_{j}\right) f_{3}\left(\mathbf{x}_{j}\right)
$$

Generalized force

$\mathbf{Q}_{T}\left(\mathbf{x}_{j}\right)=\frac{\partial \mathbf{\Psi}_{r 3}^{T}\left(\mathbf{x}_{j}\right)}{\partial x_{2}} \tau_{1}\left(\mathbf{x}_{j}\right)$

Generalized torque

$$
\mathbf{Q}_{j}=\Psi_{v}^{T} q_{j}
$$

Generalized charge 
where $\mathbf{M}_{t i p}$ is calculated in $x_{2}=\frac{L}{2}$. The rest of the coefficients are calculated from the integral terms in (18) where the first integral gives the mass matrix for the beam.

$$
\mathbf{M}_{s}=\int_{V_{s}}-\rho_{s} \boldsymbol{\Psi}_{r}^{T} \boldsymbol{\Psi}_{r}=-\rho_{s} A_{s} \int_{0}^{L} \boldsymbol{\Psi}_{r 3}^{T} \mathbf{\Psi}_{r 3}-\rho_{s} I_{s} \frac{\partial \mathbf{\Psi}_{r 3}^{T}}{\partial x_{2}} \frac{\partial \mathbf{\Psi}_{r 3}}{\partial x_{2}} d x_{2}
$$

with $A_{s}$ and $I_{s}$ defined in equation (33). The last integral expresses rotational energy, however, since it only represents a fraction of the first integral it can be neglected. A calculation shows that the 2 . mode rotational energy is a factor $5 \cdot 10^{-4}$ smaller than the corresponding translational energy. The second integral term in equation (18) gives the mass matrix for the piezoelements

$$
\mathbf{M}_{p}=\int_{V_{p}}-\rho_{p} \boldsymbol{\Psi}_{r}^{T} \boldsymbol{\Psi}_{r}=-\rho_{p} A_{p} \sum_{i=1}^{2} \int_{a_{i 1}}^{a_{i 2}} \boldsymbol{\Psi}_{r 3}^{T} \boldsymbol{\Psi}_{r 3}-\rho_{p} I_{p} \frac{\partial \boldsymbol{\Psi}_{r 3}^{T}}{\partial x_{2}} \frac{\partial \boldsymbol{\Psi}_{r 3}}{\partial x_{2}} d x_{2}
$$

Again the last term which represents the rotational mass can be neglected. The third integral represents the stiffness of the beam

$$
\mathbf{K}_{s}=\int_{V_{s}}\left(\mathbf{L}_{u} \boldsymbol{\Psi}_{r}\right)^{T} \mathbf{c}_{s} \mathbf{L}_{u} \boldsymbol{\Psi}_{r}=I_{s} \mathbf{b}_{s} \mathbf{c}_{s} \mathbf{b}_{s}^{T} \int_{0}^{L} \frac{\partial^{2} \mathbf{\Psi}_{r 3}^{T}}{\partial x_{2}^{2}} \frac{\partial^{2} \boldsymbol{\Psi}_{r 3}}{\partial x_{2}^{2}} d x_{2}
$$

The stiffness matrix representing the piezoelements is calculated form the fifth integral

$$
\mathbf{K}_{p}=\int_{V_{p}}\left(\mathbf{L}_{u} \boldsymbol{\Psi}_{r}\right)^{T} \mathbf{c}^{E} \mathbf{L}_{u} \boldsymbol{\Psi}_{r} \quad=I_{p} \mathbf{b}_{p} \mathbf{c}^{E} \mathbf{b}_{p}^{T} \sum_{i=1}^{2} \int_{a_{i 1}}^{a_{i 2}} \frac{\partial^{2} \boldsymbol{\Psi}_{r 3}^{T}}{\partial x_{2}^{2}} \frac{\partial^{2} \boldsymbol{\Psi}_{r 3}}{\partial x_{2}^{2}} d x_{2}
$$

The fourth integral represents the electro-mechanical coupling

$$
\begin{aligned}
\boldsymbol{\Gamma} & =\int_{V_{p}}\left(\mathbf{L}_{u} \boldsymbol{\Psi}_{r}\right)^{T} \mathbf{e}^{T} \mathbf{L}_{\varphi} \boldsymbol{\Psi}_{v} \\
& =-w_{s}\left(t_{p}^{2}+t_{s} t_{p}\right)\left(v_{p} e_{33}-e_{31}\right) \sum_{i=1}^{2} \int_{a_{i 1}}^{a_{i 2}} \frac{\partial^{2} \boldsymbol{\Psi}_{r 3}^{T}}{\partial x_{2}^{2}}\left[\frac{\delta_{i 1}}{t_{p}} \frac{\delta_{i 2}}{t_{p}}\right] d x_{2}
\end{aligned}
$$

where $\delta_{i j}$ is the Kronecker delta. The sixth integral term is an expression for the capacitance of the piezoelements

$$
\mathbf{C}_{p}=2 w_{s} \varepsilon_{3}^{S}\left[\begin{array}{cc}
\frac{a_{12}-a_{11}}{t_{p}} & 0 \\
0 & \frac{a_{22}-a_{21}}{t_{p}}
\end{array}\right]
$$

The seventh integral represents the mechanical-electro coupling and is seen to be identical to the transpose of the electromechanical coupling. In the equations above the following notations has been used

$$
\begin{aligned}
& A_{s}=w_{s} t_{s}, A_{p}=w_{s} t_{p}, I_{s}=\frac{1}{12} w_{s} t_{s}^{3}, I_{p}=\frac{2}{3} w_{s}\left(3 t_{p}^{2} \frac{t_{s}}{2}+3 \frac{t_{s}^{2}}{4} t_{p}+t_{p}^{3}\right) \\
& \mathbf{b}_{s}=\left[\begin{array}{llllll}
0 & -1 & v_{s} & 0 & 0 & 0
\end{array}\right], \quad \mathbf{b}_{p}=\left[\begin{array}{llllll}
0 & -1 & v_{p} & 0 & 0 & 0
\end{array}\right]
\end{aligned}
$$

Equations (19) and (20) are denoted as actuator and sensor equations since the actuator equation gives the mechanical displacements when the voltages at the piezoelements are prescribed whereas the sensor equation gives the voltages at the open circuted electrodes of the piezoelements for a given displacement
Table 1. RESONANCE FREQUENCIES FOR THE TWO PLACEMENTS OF PIEZOELEMENTS SHOWN IN FIGURE 3.

\begin{tabular}{|l|c|c|c|c|c|c|}
\hline & $\Gamma_{11}$ & $\Gamma_{21}$ & $\Gamma_{31}$ & $f_{1}[k H z]$ & $f_{2}[k H z]$ & $\frac{f_{2}}{f_{1}}$ \\
\hline Placement 1 & -0.016 & -0.057 & 0.006 & 2.02 & 5.38 & 2.67 \\
\hline Placement 2 & 0.021 & 0.027 & 0.005 & 3.21 & 5.46 & 1.71 \\
\hline
\end{tabular}

of the resonator. Here the actuator equation is of most importance. To include damping in the resonator a damping matrix $\mathbf{C}$ is introduced in the actuator equation

$$
\mathbf{M} \ddot{\mathbf{r}}+\mathbf{C} \dot{\mathbf{r}}+\mathbf{K r}-\mathbf{\Gamma} \mathbf{v}=\sum_{j=1}^{n_{f}} \mathbf{Q}_{F}\left(\mathbf{x}_{j}\right)+\sum_{j=1}^{n_{t}} \mathbf{Q}_{T}\left(\mathbf{x}_{j}\right)
$$

where the damping matrix can are defined as $\mathbf{C}=\alpha \mathbf{M}+\beta \mathbf{K}$. The coefficients used are $\alpha=7.5$ and $\beta=2 \cdot 10^{-5}$.

\section{Optimum Placement of Piezoelements}

The electro-mechanical coupling term $\boldsymbol{\Gamma}$ indicates how effective the voltages $v_{1}(t)$ and $v_{2}(t)$ excites each mode-shape since the elements of $\Gamma_{i j}$ are proportional to the elements of $\frac{\partial^{2} u_{3_{i}}}{\partial x_{2}^{2}}$ integrated over the length of the piezoelements. Therefore the values of $\Gamma_{11}, \Gamma_{12}, \Gamma_{21}$ and $\Gamma_{22}$ should be maximised with respect to the rest of the elements of $\boldsymbol{\Gamma}$. In Figure 3 the first five second derivatives of the mode shapes are shown and the positions of the piezoelements are marked with a bold line. To see how the placement of the piezoelements affects the resonance frequencies two solutions are shown in Figure 3, that is, the placements marked 1 and 2. The values of the first three components of $\boldsymbol{\Gamma}$ in the first column and the first two resonance frequencies are shown in Table 1. The two different ratios $\frac{f_{2}}{f_{1}}$ shows that it is possible to change the ratio to an optimal value by changing the position and size of the piezoelements. Also Table 1 shows that $\Gamma_{31}$ has been minimized compared to $\Gamma_{11}$ and $\Gamma_{21}$ although it is not reduced to zero. Figure 4 shows simulations of the tip trajectories using the two different placements of the piezoelements. Figures $4 a-b$ show simulations for placement 2 where the excitation frequencies are $\omega_{1}=\pi f_{2}$ and $\omega_{2}=2 \pi f_{2}$ for figure a, and $\omega_{1}=2 \pi f_{1}$ and $\omega_{2}=4 \pi f_{1}$ in figure $\mathrm{b}$. The number " 8 " is slightly wider when the second mode excitation frequency equals the second mode resonance frequency and similarly is the trajectory higher when the first mode excitation frequency equals the first mode resonance frequency. Simulations for placement 1 of the piezoelements are shown in figures $4 c-d$ where the excitation frequencies are equal to the above figures. Here the hight of the trajectory is clearly degraded when the first vibration mode is not excited in it's resonance frequency. Therefore placement 2 seems to be the best choice and this configuration will be used in the rest of the pa- 


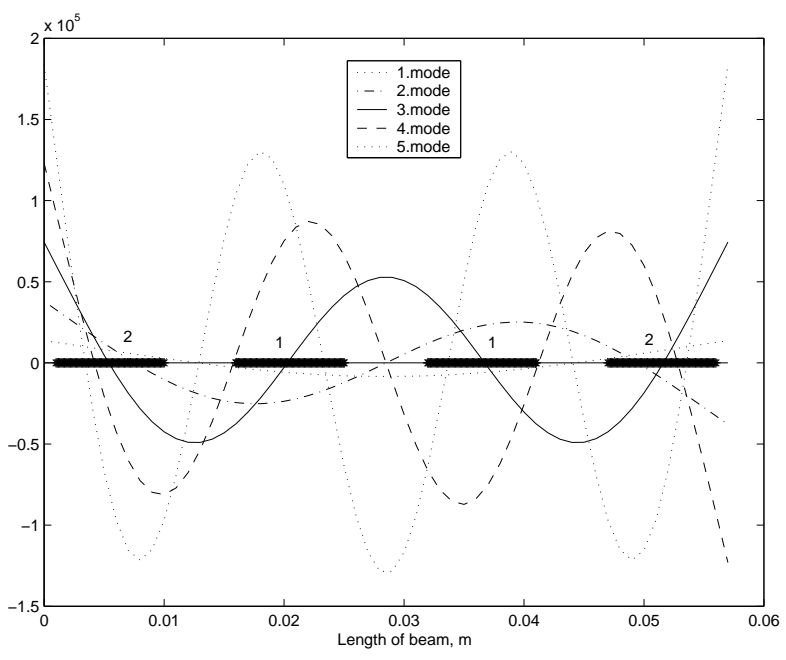

Figure 3. OPTIMUM PLACEMENT OF PIEZOELEMENTS.
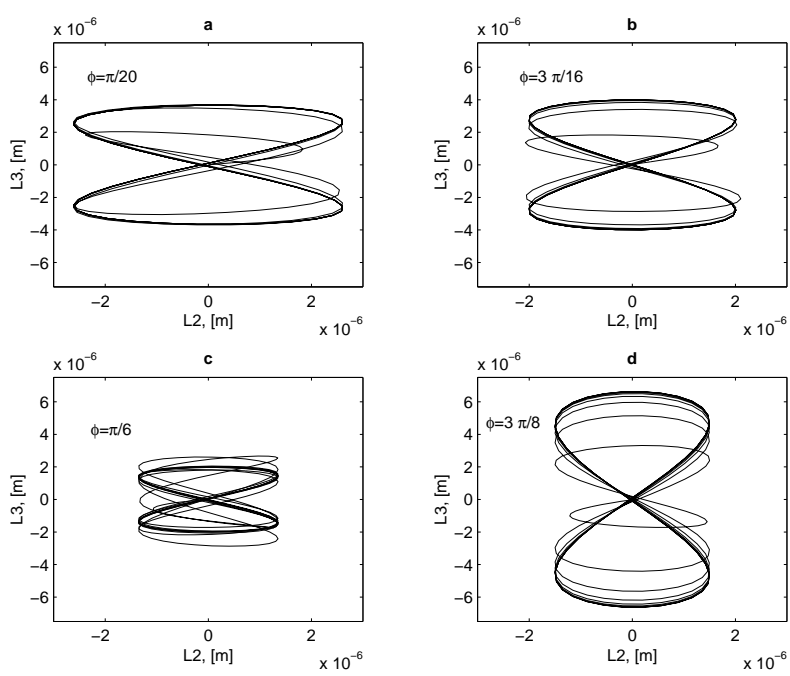

Figure 4. SIMULATION OF TIP TRAJECTORIES FOR THE TWO CONFIGURATIONS OF THE RESONATOR.

per. To obtain symmetric trajectories it is necessary to adjust the phase $\phi$ in each individual case. The phase values obtained by trial and error are written in each figure. The equations describing the trajectories of the position of the tip-end are given by

$\mathbf{L}=\left[\begin{array}{l}L_{2}\left(r_{1}, \ldots, r_{n}\right) \\ L_{3}\left(r_{1}, \ldots, r_{n}\right)\end{array}\right]=\left[\begin{array}{c}-L_{t i p} \sin \theta \\ L_{t i p} \cos \theta+u_{3}\end{array}\right]=\left[\begin{array}{c}-L_{t i p} \sin \theta \\ L_{t i p} \cos \theta+\mathbf{\Psi}_{r 3} \mathbf{r}\end{array}\right]$

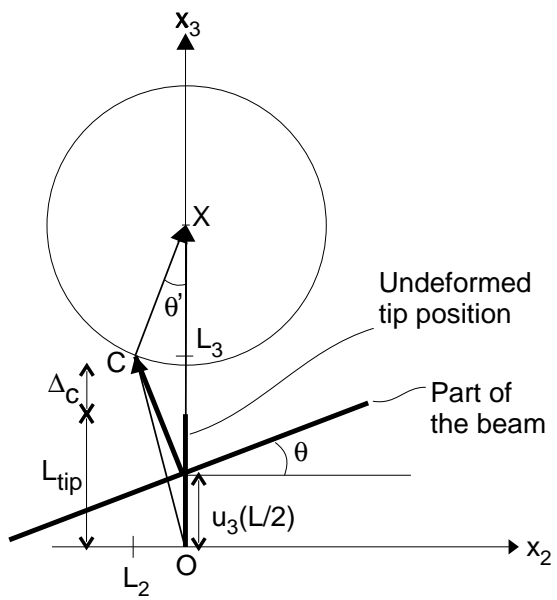

Figure 5. CALCULATION OF THE CONSTRAINT FROM THE STATOR AND ROTOR GEOMETRY.

\section{CONTACT MODELLING \\ Expression for the Constraint}

The motion of the constrained tip can be described with the vector $\mathbf{O C}$ in Figure 5. The origin of the coordinate system is defined in the centre of the undeformed beam, $\Delta_{c}$ is the initial clearance between rotor and tip. $R$ is the radius of the rotor. The three vectors in Figure 5 give the equation

$$
\mathbf{C X}=\mathbf{O X}-\mathbf{O C}=\mathbf{O X}-\mathbf{L}
$$

Using equation (35) and calculating the squared magnitudes on both sides of equation (36) and equating to zero gives the constraint equation

$$
\begin{aligned}
C\left(r_{1}, \ldots, r_{n}\right) & =|\mathbf{O X}-\mathbf{O C}|^{2}-R^{2} \\
& =L_{2}^{2}+L_{3}^{2}+\left(L_{t i p}+\Delta_{c}+R\right)^{2}-2\left(L_{t i p}+\Delta_{c}+R\right) L_{3}-R^{2}=0 .
\end{aligned}
$$

To incorporate the constraint in the equation for the free vibrating resonator the variation of the constraint is taken with respect to the generalized coordinates and the expression is multiplied with a factor in accordance with the Lagrange multiplier method. Using variational calculus the constraint term in Hamilton's equation can be expressed as

$$
\lambda \delta C\left(r_{1}, \cdots, r_{n}\right)=\lambda \frac{\partial C}{\partial \mathbf{r}} \delta \mathbf{r}
$$


where the derivative of $C\left(r_{1}, \cdots, r_{n}\right)$ gives

$$
\begin{aligned}
\frac{\partial C}{\partial \mathbf{r}}= & \frac{\partial C}{\partial \mathbf{L}} \frac{\partial \mathbf{L}}{\partial \mathbf{r}} \\
= & {\left[\begin{array}{lll}
-2 L_{t i p} \sin \theta & 2\left(L_{t i p} \cos \theta+\mathbf{\Psi}_{r 3} \mathbf{r}\right)-2\left(L_{t i p}+\Delta_{C}+R\right)
\end{array}\right] } \\
& {\left[\begin{array}{ccc}
-L_{t i p} \cos \theta \frac{\partial u_{3_{1}}}{\partial x_{2}} & \cdots & -L_{t i p} \cos \theta \frac{\partial u_{3_{n}}}{\partial x_{2}} \\
-L_{t i p} \sin \theta \frac{\partial u_{3_{1}}}{\partial x_{2}}+u_{3_{1}} & \cdots & -L_{t i p} \sin \theta \frac{\partial u_{3_{n}}}{\partial x_{2}}+u_{3_{n}}
\end{array}\right] }
\end{aligned}
$$

Equation (38) can be formulated in terms of variations in $L_{2}$ and $L_{3}$ using variational calculus

$$
\lambda \frac{\partial C}{\partial \mathbf{L}} \frac{\partial \mathbf{L}}{\partial \mathbf{r}} \delta \mathbf{r}=\lambda \frac{\partial C}{\partial \mathbf{L}} \sum_{i} \frac{\partial \mathbf{L}}{\partial r_{i}} \delta r_{i}=\lambda \frac{\partial C}{\partial \mathbf{L}} \delta \mathbf{L}
$$

Since the variation of the last expression is taken with respect to the physical displacements $L_{2}$ and $L_{3}$ this shows that $\lambda \frac{\partial C}{\partial \mathrm{L}}$ is the physical normal force due to the constraint. The direction of $\frac{\partial C}{\partial \mathbf{L}}$ is normal to the surface of the rotor which can be shown geometrically from Figure 6a since the components of $\frac{1}{2} \frac{\partial C}{\partial \mathbf{L}}$ forms the vector XC. During contact the normal force acting on the tip should have the direction of $\frac{\partial C}{\partial \mathrm{L}}$, therefore the sign of $\lambda$ must be positive during contact. When the tip loses contact with the rotor the sign of $\lambda$ will change as a consequence of the constraint equation. The expression for the generalized normal force is

$$
\mathbf{Q}_{N}=\lambda \frac{\partial C}{\partial \mathbf{L}} \frac{\partial \mathbf{L}}{\partial \mathbf{r}}=\mathbf{F}_{N} \frac{\partial \mathbf{L}}{\partial \mathbf{r}}
$$

where $\mathbf{F}_{N}$ is the physical normal force. Rewriting equation (39) shows that the generalised normal force can be decomposed into magnitudes of the physical forces $\mathbf{F}_{\tau}$ and $\mathbf{F}_{3}$ multiplied with $L_{t i p} \frac{\partial \Psi_{3}}{\partial x_{2}}$ and $\Psi_{3}$, respectively

$$
\begin{aligned}
\lambda \frac{\partial C}{\partial \mathbf{r}} & =\lambda \frac{\partial C}{\partial \mathbf{L}}\left[\begin{array}{l}
-\cos \theta \\
-\sin \theta
\end{array}\right] L_{t i p} \frac{\partial \Psi_{r 3}}{\partial x_{2}}+\lambda \frac{\partial C}{\partial \mathbf{L}}\left[\begin{array}{l}
0 \\
1
\end{array}\right] \boldsymbol{\Psi}_{r 3} \\
& =F_{\tau} L_{t i p} \frac{\partial \boldsymbol{\Psi}_{r 3}}{\partial x_{2}}+F_{3} \boldsymbol{\Psi}_{r 3}
\end{aligned}
$$

Figure 6 b shows $\mathbf{F}_{\tau}$ and $\mathbf{F}_{3}$ as projections of the normal force on the unit vectors $\left[\begin{array}{l}-\cos \theta \\ -\sin \theta\end{array}\right]$ and $\left[\begin{array}{l}0 \\ 1\end{array}\right]$. This decomposition is a little peculiar since the unit vectors are not orthogonal. However, as long as $\theta$ is small the error introduced using the non-orthogonal projection vectors is small. Comparing equation (42) with the equations for applied torque and force (24) and (25) also shows the resemblance between torque and force expressions; torques are multiplied with $\frac{\partial \Psi_{r 3}}{\partial x_{2}}$ to form generalized torques and forces are multiplied with $\boldsymbol{\Psi}_{r 3}$ to forme generalized forces.

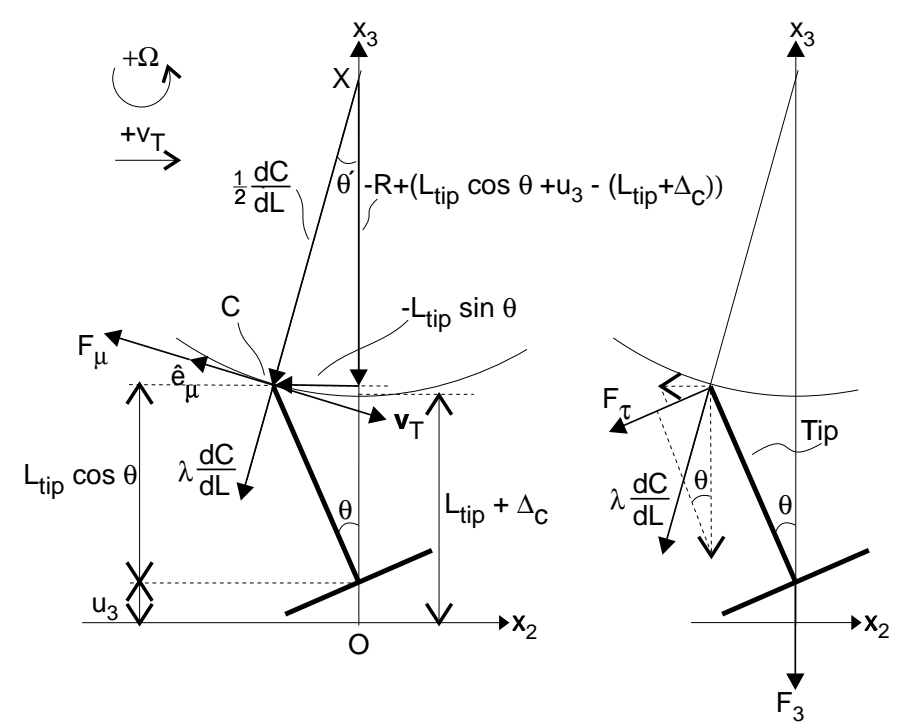

Figure 6. FIG. a, THE NORMAL FORCE $\lambda \frac{\partial C}{\partial \mathrm{L}}$ IS NORMAL TO THE ROTOR AND THE FRICTION FORCE IS PARALLEL WITH $\hat{\mathbf{e}}_{\mu}$. FIG. b, INTERPRETATION OF FORCES FROM THE LAGRANGE MULTIPLIER.

\section{Friction Force}

The friction force vector is shown on Figure $6 \mathrm{a}$ and has the expression

$$
\mathbf{F}_{\mu}=\operatorname{sign}\left(v_{T}-\Omega R\right) \mu|\lambda|\left|\frac{\partial C}{\partial \mathbf{L}}\right| \hat{\mathbf{e}}_{\mu}
$$

where the Coulomb friction law is assumed, $\mu$ is the coefficient of friction and $\hat{\mathbf{e}}_{\mu}$ is the unit vector $\left[\begin{array}{c}-\cos \theta^{\prime} \\ \sin \theta^{\prime}\end{array}\right]$ and $v_{T}$ is the velocity of the tip during contact

$$
v_{T}=-\mathbf{v}_{T} \hat{\mathbf{e}}_{\mu}, \quad \mathbf{v}_{T}=\dot{\mathbf{L}}=\left(\left[\begin{array}{c}
-\cos \theta \\
-\sin \theta
\end{array}\right] L_{t i p} \frac{\partial \boldsymbol{\Psi}_{r 3}}{\partial x_{2}}+\left[\begin{array}{l}
0 \\
1
\end{array}\right] \boldsymbol{\Psi}_{r 3}\right) \dot{\mathbf{r}}
$$

With the sign definition of $\mathbf{F}_{\mu}$ given in equation (43) and the definition of positive directions for $v_{T}$ and $\Omega$ as indicated on Figure 6a the signs of $\operatorname{sign}\left(v_{T}-\Omega R\right)$ and direction of $\mathbf{F}_{\mu}$ for all possible relative velocities between rotor and tip is shown in Table 2. To form the generalized friction force it follows from equation (41) that $\mathbf{F}_{\mu}$ should be multiplied with $\frac{\partial \mathrm{L}}{\partial \mathbf{r}}$

$$
\mathbf{Q}_{\mu}=\operatorname{sign}\left(v_{T}-\Omega R\right) \mu|\lambda|\left|\frac{\partial C}{\partial \mathbf{L}}\right| \hat{\mathbf{e}}_{\mu} \frac{\partial \mathbf{L}}{\partial \mathbf{r}}
$$

where the friction force vector is projected onto the nonorthogonal unit vectors as shown in equation (42) The unit vector 
Table 2. SIGN OF $\left(v_{T}-\Omega R\right)$ AND DIRECTION OF $\mathbf{F}_{\mu}$.

\begin{tabular}{|c|c|c|c|c|c|}
\cline { 3 - 6 } \multicolumn{2}{c|}{} & \multicolumn{2}{c|}{$v_{T}$ positive } & \multicolumn{2}{c|}{$v_{T}$ negative } \\
\cline { 3 - 6 } \multicolumn{2}{c|}{} & $v_{T}>\Omega R$ & $v_{T}<\Omega R$ & $v_{T}>\Omega R$ & $v_{T}<\Omega R$ \\
\hline$\Omega R>0$ & $\operatorname{sign}\left(v_{T}-\Omega R\right)$ & + & - & & - \\
\cline { 2 - 6 } & Direction of $\mathbf{F}_{\mu}$ & left & right & & right \\
\hline \multirow{2}{*}{$\Omega R<0$} & $\operatorname{sign}\left(v_{T}-\Omega R\right)$ & + & & + & - \\
\cline { 2 - 5 } & Direction of $\mathbf{F}_{\mu}$ & left & & left & right \\
\hline
\end{tabular}

$\hat{\mathbf{e}}_{\mu}$ can also be expressed using $\frac{\partial C}{\partial \mathbf{L}}$

$$
\hat{\mathbf{e}}_{\mu}=\frac{1}{\left|\frac{\partial C}{\partial \mathbf{L}}\right|} \frac{\partial C}{\partial \mathbf{L}}\left[\begin{array}{cc}
0 & -1 \\
1 & 0
\end{array}\right]
$$

Therefore the generalised friction force is reduced to

$$
\mathbf{Q}_{\mu}=\operatorname{sign}\left(v_{T}-\Omega R\right) \mu|\lambda| \frac{\partial C}{\partial \mathbf{L}}\left[\begin{array}{cc}
0 & -1 \\
1 & 0
\end{array}\right] \frac{\partial \mathbf{L}}{\partial \mathbf{r}}=|\lambda| \mathbf{Q}_{\mu}^{*}
$$

with,

$$
\mathbf{Q}_{\mu}^{*}=\operatorname{sign}\left(v_{T}-\Omega R\right) \mu \frac{\partial C}{\partial \mathbf{L}}\left[\begin{array}{cc}
0 & -1 \\
1 & 0
\end{array}\right] \frac{\partial \mathbf{L}}{\partial \mathbf{r}}
$$

The friction force is included in the rotor model as follows

$$
J \dot{\Omega}+C_{r} \Omega=\operatorname{sign}\left(v_{T}-\Omega R\right)\left|\mathbf{F}_{\mu}\right|-\tau_{a p p}
$$

where $\Omega$ is the angular velocity, $C_{r}$ is a viscous damping coefficient, $\tau_{a p p}$ is an applied torque and $J$ is the moment of inertia.

\section{SOLUTION TO THE SYSTEM}

The equations describing the constrained motion in the state of slip are

$$
\mathbf{M} \ddot{\mathbf{r}}+\mathbf{C} \dot{\mathbf{r}}+\mathbf{K r}=\mathbf{\Gamma v}+\mathbf{Q}_{N}^{T}+\mathbf{Q}_{\mu}^{T}, \quad C(\mathbf{r})=0,
$$

Equations (49) forms a set of differential-algebraic equations (DAEs) which has an index equal to 3 . The index of a DAE is defined as the minimal number of times that all or part of the equations must be differentiated with respect to time, in order to determine the derivatives of all variables in the DAE (Yen et al. 1991), that is to determine $\dot{\mathbf{r}}$ and $\dot{\lambda}$ in terms of $t$ and $\mathbf{r}$. The index provides information about the numerical and analytical difficulties in solving the equations (Campbell and Leimkuhler 1991). The method used here which was proposed by (Gear et al. 1985) reduces the index of the original system to an index 2 problem. The method which consists of a predictor-corrector integrator can be summarised as follows. follows

Predictor: The constraint equation is differentiated twice as

$$
\frac{\partial^{2} C(\mathbf{r})}{\partial t^{2}}=\dot{\mathbf{r}}^{T} \frac{\partial^{2} C}{\partial \mathbf{r}^{2}} \dot{\mathbf{r}}+\frac{\partial C}{\partial \mathbf{r}} \ddot{\mathbf{r}}=\dot{\mathbf{r}}^{T} \frac{\partial \mathbf{G}}{\partial \mathbf{r}} \dot{\mathbf{r}}+\mathbf{G} \ddot{\mathbf{r}}=0
$$

with $\mathbf{G}=\frac{\partial C}{\partial \mathbf{r}}$. Solving for $\ddot{\mathbf{r}}$ in (49) and substituting in (50) gives

$\lambda \mathbf{G M}^{-1} \mathbf{G}^{T}+|\lambda| \mu \mathbf{G M}^{-1} \mathbf{Q}_{\mu}^{*}=\mathbf{G M}^{-1}(\mathbf{C} \dot{\mathbf{r}}+\mathbf{K r}-\Theta \mathbf{v})-\dot{\mathbf{r}}^{T} \frac{\partial \mathbf{G}}{\partial \mathbf{r}} \dot{\mathbf{r}}$.

Equation (51) can be solved for $\lambda$ if it's sign is known. Since the sign of $\lambda$ is the same for a system with friction and a frictionless system the sign can be determined when the friction term in equation (51) is neglected. With the known sign the correct magnitude of $\lambda$ is calculated afterwards from equation (51) and with this value $\mathbf{r}$ can be calculated from equation (49). For a correct solution $C(\mathbf{r})$ must equal zero during contact but using this method only $\ddot{C}$ is guaranteed to equal zero. Even with initial values $C\left(\mathbf{r}\left(t_{0}\right)\right)=0$ and $\dot{C}\left(\mathbf{r}\left(t_{0}\right)\right)=0$ the solution will drift due to numerical errors (Hatman et al. 1996). Therefore equations (51) and (49) are used to predict values of $\lambda$ and generalised coordinates and velocities.

Corrector: A new system of equations is constructed with index two, where solutions of this new system are solutions of the original system of equations. Denoting the generalised velocities with $\mathbf{q}$ the system of equations to be solved are

$$
\mathbf{q}=\dot{\mathbf{r}}, \quad \mathbf{M} \dot{\mathbf{q}}+\mathbf{C q}+\mathbf{K r}=\mathbf{\Theta} \mathbf{v}+\lambda \mathbf{G}^{T}+|\lambda| \mathbf{Q}_{\mu}^{* T}, \quad C(\mathbf{r})=0
$$

The system is reduced to an index two system given by

$$
\begin{aligned}
& \dot{\mathbf{r}}=\mathbf{q}+\kappa \mathbf{G} \\
& \mathbf{M} \dot{\mathbf{q}}+\mathbf{C q}+\mathbf{K r}=\mathbf{\Theta} \mathbf{v}+\lambda \mathbf{G}^{T}+|\lambda| \mathbf{Q}_{\mu}^{* T} \\
& C(\mathbf{r})=0 \\
& \frac{\partial C}{\partial t}=\frac{\partial C}{\partial \mathbf{r}} \frac{\partial \mathbf{r}}{\partial t}=\mathbf{G q}=0
\end{aligned}
$$

The steps necessary to solve this system during contact are

Step 1: Predict $\lambda_{n}^{\text {pred }}, \mathbf{r}_{n}^{\text {pred }}, \mathbf{q}_{n}^{\text {pred }}$ using equations (49) and (51), where $t_{n}$ is the time index.

Step 2: Correct $\mathbf{r}_{n}^{\text {pred }}$, by solving equations (53) and (55) with $\kappa=0$. Using a backward differentiation method (BDF) in 
combination with a gradient method gives

$$
\mathbf{r}_{n}=\mathbf{r}_{n-1}+t_{\text {step }} \mathbf{q}_{n}^{\text {pred }}-\frac{C\left(\mathbf{r}_{n}^{\text {pred }}\right)}{\mathbf{G}\left(\mathbf{r}_{n}^{\text {pred }}\right) \mathbf{G}\left(\mathbf{r}_{n}^{\text {pred }}\right)^{T}} \mathbf{G}\left(\mathbf{r}_{n}^{\text {pred }}\right)
$$

Step 3: Using the same BDF formula equation (54) is used to solve for the corrected generalised velocities,

$$
\begin{aligned}
& \mathbf{q}_{n}=\mathbf{q}_{n-1}+ \\
& h \mathbf{M}^{-1}\left(-\mathbf{C} \mathbf{q}_{n}^{\text {pred }}-\mathbf{K} \mathbf{r}_{n}+\Theta \mathbf{v}_{n}+\lambda_{n} \mathbf{G}\left(\mathbf{r}_{n}\right)^{T}+\left|\lambda_{n}\right| \mathbf{Q}_{\mu}^{*}\left(\mathbf{r}_{n}\right)^{T}\right)
\end{aligned}
$$

where $\lambda_{n}$ is solved by substituting the above expression for $\mathbf{q}_{n}$ in equation (56).

$$
\begin{aligned}
& t_{\text {step }} \mathbf{G}\left(\mathbf{r}_{n}\right) \mathbf{M}^{-1}\left(\lambda_{n} \mathbf{G}\left(\mathbf{r}_{n}\right)^{T}+\left|\lambda_{n}\right| \mathbf{Q}_{\mu}^{*}\left(\mathbf{r}_{n}\right)^{T}\right)= \\
& -\mathbf{G}\left(\mathbf{r}_{n}\right) \mathbf{q}_{n-1}+t_{\text {step }} \mathbf{G}\left(\mathbf{r}_{n}\right) \mathbf{q}_{n-1} \mathbf{M}^{-1}\left(\mathbf{C} \mathbf{q}_{n}^{\text {pred }}+\mathbf{K} \mathbf{r}_{n}-\Theta \mathbf{v}_{n}\right) .
\end{aligned}
$$

This equation is solved for $\lambda_{n}$ using the same procedure as in equation (51).

\section{Algorithm for the Solution}

The algorithm for solving the system equations consists of two loops, one for the unconstrained motion and one for the constrained motion. In the unconstrained motion loop the next values of generalised coordinates and velocities are calculated with a Runge-Kutta method and the clearance between tip and rotor is calculated. Now, depending on this clearance two possibilities exists:

1. If the clearance is positive the values (values for coordinates, velocities, time, $\lambda$, and forces) are updated and the unconstrained motion loop is continued.

2. If the clearance is negative or zero the time $t_{\text {zero }}$ when the clearance equals zero is calculated with a linear interpolation. The values for coordinates and velocities at $t_{z e r o}$ are also calculated with a linear interpolation. Based on these values new coordinates, velocities and a new clearance is calculated.

If the new clearance calculated in step 2 is negative the unconstrained motion loop is terminated and the evaluation of tip motion is continued in the loop for constrained motion. In this loop first the time step-size is set to a small value. Then a prediction is made for next values of coordinates, velocities and $\lambda$ and a correction of these values. Depending on the value of $\lambda$ two possibilities exists:
Table 3. DIFFERENT DIMENSIONS OF THE MOTOR.

\begin{tabular}{|l|l|l|l|l|l|}
\hline$t_{s}[\mathrm{~m}]$ & $t_{p}[\mathrm{~m}]$ & $w_{s}[\mathrm{~m}]$ & $L_{\text {tip }}[\mathrm{m}]$ & $L[\mathrm{~m}]$ & $R[\mathrm{~m}]$ \\
\hline $1 \cdot 10^{-3}$ & $1 \cdot 10^{-3}$ & $16 \cdot 10^{-3}$ & $19 \cdot 10^{-3}$ & $57 \cdot 10^{-3}$ & $25 \cdot 10^{-3}$ \\
\hline
\end{tabular}

1. If $\lambda$ is positive the values are updated and the constrained motion loop is continued.

2. If $\lambda$ is negative or zero the time $t_{\text {zero }}$ when $\lambda=0$ is calculated with a linear interpolation. The values for coordinates and velocities at $t_{\text {zero }}$ are also calculated with a linear interpolation. Since the sign of $\lambda$ has changed from positive to negative this means that the tip is loosing contact with the rotor and the clearance should change to some positive value. However simulations show that during the constrained motion the negative value of the clearance increases, therefore it is necessary to perform a number of unconstrained calculations of the tip motion until the sign of the clearance becomes positive, before entering into the unconstrained loop.

\section{SIMULATION RESULTS}

The dimensions for the motor used in the simulation are given in Table 3. The moment of inertia for the disk is $J=$ $4.8 \cdot 10^{-5} \mathrm{~kg} \mathrm{~m}^{2}$ and the applied voltages are $U_{1}=U_{2}=100 \mathrm{~V}$ The material used for the beam is steel and the piezoceramic is PZ26. All simulations has been carried out using the first four eigenmodes of the Bernoulli-Euler beam. Figure 7 shows the trajectories of unconstrained motion in figure a, and constrained motion of the tip in figures b-d for initial clearance equal to $0 \mu m, 1 \mu m$ and $2 \mu m$. It was found by trial and error that a change of the phase in equation (2) to $\phi=-\frac{\pi}{5}$ gave the highest rotor accelerations (but not the highest final velocity). Figure 8 a shows the rotor velocities corresponding to the trajectories in Figure 7c-d, where $C_{r}=0$ and $\tau_{a p p}=0$ has been used. The dotted line shows the change in velocity during the impact time step. The solid line indicate the rest of the contact period, whereas the dashed line indicates nocontact. It is seen that the impact is responsible for a major part of the rotor acceleration due to the high impact forces. The case with initial clearance $\Delta_{C}=0 \mu m$ shows the best performance due to a longer contact period. The results indicates that the performance may improve if an initial pressure between tip and rotor is provided. The steady state velocity is shown in Figure $8 \mathrm{~b}$ where the initial angular velocity was prescribed to $-0.9 \frac{\mathrm{rad}}{\mathrm{s}}$. The long de-acceleration period consists of de-accelerations caused by an impact followed by an acceleration and de-acceleration during the contact. To see the torque performance of the motor equation (48) is solved with values of $\tau_{a p p}$ equal to $0 \mathrm{Nm}, 0.001 \mathrm{Nm}$, $0.002 \mathrm{Nm}$ and $0.005 \mathrm{Nm}$. The results are shown in Figure 8c.

Figure 9 shows the normal force acting on the tip. The 

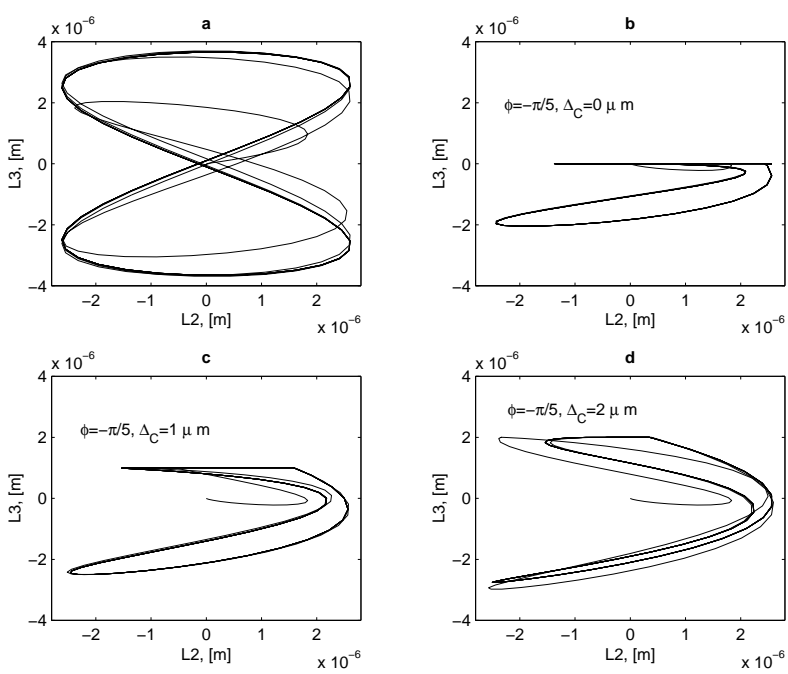

Figure 7. FIG. a, VIBRATION. FIG. b-c, CONSTRAINED TIP TRAJECTORIES.
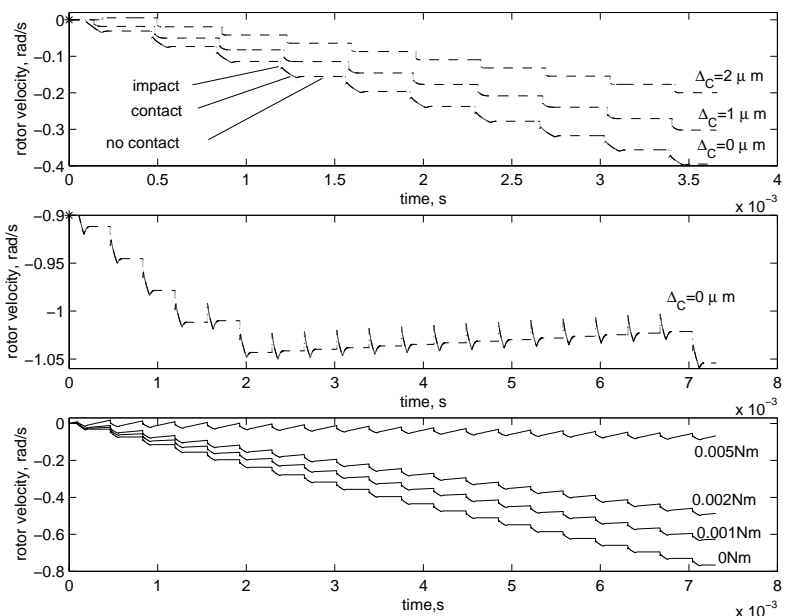

Figure 8. FIG. a, TRANSIENT VELOCITIES AT THREE INITIAL CLEARANCE VALUES. FIG. b, STEADY STATE VELOCITY. FIG. c, TORQUE-VELOCITY SIMULATIONS.

high peaks of normal force is caused by the Lagrange Multiplier method which forces the normal tip-velocity to be de-accelerated to zero during one time step. This generates a high normal force that has no direct physical meaning, however, the resulting rotor velocity do have physical meaning since it corresponds to the time integral of the friction force which is proportional to the normal force. To generate physical forces it is necessary to in-

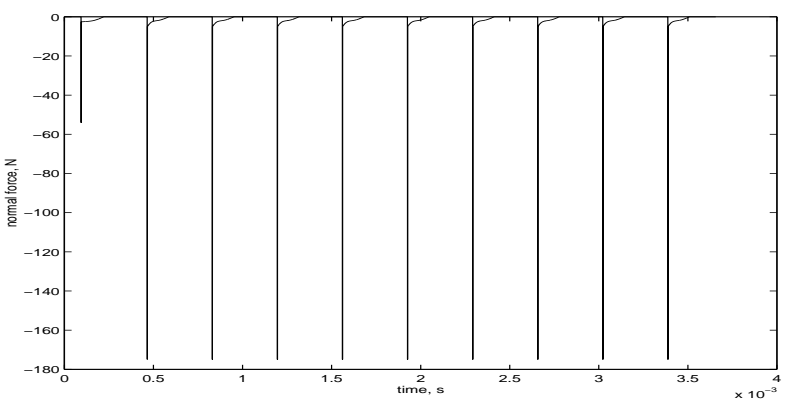

Figure 9. EXAMPLE OF NORMAL FORCE.
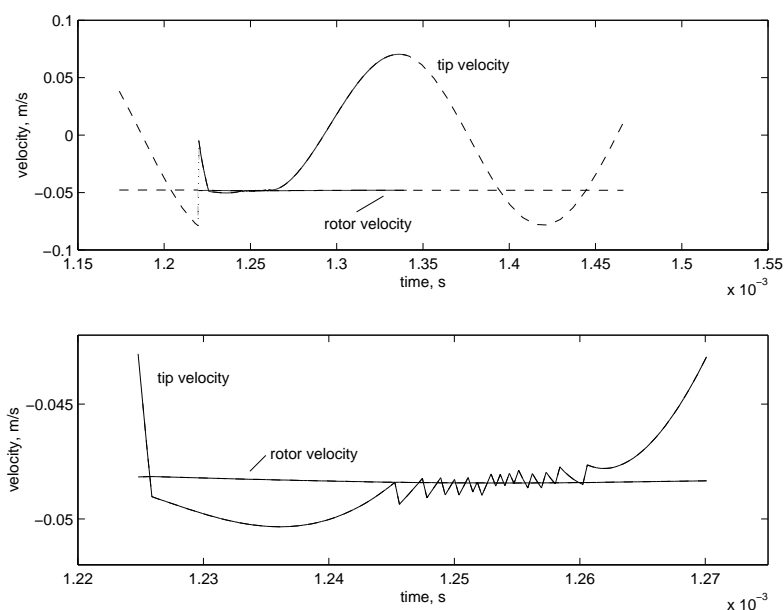

Figure 10. FIG. a, EXAMPLE OF STICKING. FIG. b, ZOOM OF THE STICK PERIOD.

clude the contact stiffness of the tip and rotor i.e. by using Hertz theory as suggested by (Zharii 1996).

\section{Transition From Acceleration to De-acceleration}

The transition from acceleration to de-acceleration of the rotor implies that stick between tip and rotor may occur. The duration of the stick, however, may be very short and of no importance for the performance of the motor. Figure 10a and the enlarged view in Figure 10b shows the rotor velocity and tip velocity. In Figure 10 it is seen that the velocities of rotor and tip oscillates during a small time interval indicating that the rotor velocity and the tip velocity are equal and therefore the tip sticks to the rotor. This suggest that a model for stick should be included in the contact model. A state of stick is characterised with the equations for friction force and relative rotor velocity 
and tangential velocity of the resonator tip.

$$
\begin{aligned}
& \left|\mathbf{F}_{\mu, s t i c k}\right| \leq \mu\left|\mathbf{F}_{N}\right| \\
& \Omega R=v_{T}=-\dot{\mathbf{L}}^{T} \hat{\mathbf{e}}_{\mu}
\end{aligned}
$$

The equations for the rotor becomes

$$
J \dot{\Omega}=R\left|\mathbf{F}_{\mu, s t i c k}\right|-\tau_{\text {ext }}
$$

where the damping coefficient has been neglected. Differentiating equation (61) with respect to time and substituting in equation (62) and solving for $\left|\mathbf{F}_{\mu, \text { stick }}\right|$ gives

$$
\left|\mathbf{F}_{\mu, s t i c k}\right|=-\frac{J \ddot{\mathbf{L}}^{T} \hat{\mathbf{e}}_{\mu}}{R^{2}}, \quad \text { with } \quad \ddot{\mathbf{L}}=\left[\begin{array}{c}
\dot{\mathbf{r}}^{T} \frac{\partial^{2} L_{y}}{\partial \mathbf{r}^{2}} \dot{\mathbf{r}} \\
\dot{\mathbf{r}}^{T} \frac{\partial^{2} L_{z}}{\partial \mathbf{r}^{2}} \dot{\mathbf{r}}
\end{array}\right]+\frac{\partial \mathbf{L}}{\partial \mathbf{r}} \ddot{\mathbf{r}}
$$

and $\tau_{\text {ext }}=0$. The friction force is given as the magnitude multiplied with the tangential unit vector

$$
\begin{aligned}
\mathbf{F}_{\mu, s t i c k} & =\operatorname{sign}\left(v_{T}\right)\left|\mathbf{F}_{\mu, \text { stick }}\right| \hat{\mathbf{e}}_{\mu}^{T} \\
& =-\operatorname{sign}\left(v_{T}\right) \frac{J}{R^{2}}\left(\left[\begin{array}{c}
\dot{\mathbf{r}}^{T} \frac{\partial^{2} L_{y}}{\partial \mathrm{r}^{2}} \mathbf{\mathbf { r }} \\
\dot{\mathbf{r}}^{T} \frac{\partial^{2} L_{z}}{\partial \mathbf{r}^{2}} \mathbf{\mathbf { r }}
\end{array}\right]^{T}+\ddot{\mathbf{r}}^{T}\left(\frac{\partial \mathbf{L}}{\partial \mathbf{r}}\right)^{T}\right) \hat{\mathbf{e}}_{\mu} \hat{\mathbf{e}}_{\mu}^{T}
\end{aligned}
$$

and the generalized friction force is $\mathbf{Q}_{\mu, \text { stick }}=\mathbf{F}_{\mu, \text { stick }} \frac{\partial \mathbf{L}}{\partial \mathbf{r}}$. The equations for the constrained motion of the resonator in the state of stick is

$$
\mathbf{M} \ddot{\mathbf{r}}_{\text {stick }}+\mathbf{C} \dot{\mathbf{r}}_{\text {stick }}+\mathbf{K} \mathbf{r}_{\text {stick }}=\mathbf{\Gamma v}+\mathbf{Q}_{\mu, s t i c k}^{T}+\mathbf{Q}_{N}^{T}, \quad C\left(\mathbf{r}_{\text {stick }}\right)=0
$$

Substitution of equation (64) in equation (65) gives

$$
\begin{gathered}
\mathbf{M}_{\text {stick }} \ddot{\mathbf{r}}_{\text {stick }}+\mathbf{C} \dot{\mathbf{r}}_{\text {stick }}+\mathbf{K} \mathbf{r}_{\text {stick }}= \\
\mathbf{\Gamma v}-\operatorname{sign}\left(v_{T}\right) \frac{J}{R^{2}}\left(\frac{\partial \mathbf{L}}{\partial \mathbf{r}}\right)^{T} \hat{\mathbf{e}}_{\mu} \hat{\mathbf{e}}_{\mu}^{T}\left[\begin{array}{l}
\dot{\mathbf{r}}^{T} \frac{\partial^{2} L_{y}}{\partial \mathbf{r}^{2}} \dot{\mathbf{r}} \\
\dot{\mathbf{r}}^{T} \frac{\partial^{2} L_{z}}{\partial \mathbf{r}^{2}} \dot{\mathbf{r}}
\end{array}\right]+\mathbf{Q}_{N}^{T} \\
\text { with } \mathbf{M}_{\text {stick }}=\mathbf{M}+\operatorname{sign}\left(v_{T}\right) \frac{J}{R^{2}}\left(\frac{\partial \mathbf{L}}{\partial \mathbf{r}}\right)^{T} \hat{\mathbf{e}}_{\mu} \hat{\mathbf{e}}_{\mu}^{T} \frac{\partial \mathbf{L}}{\partial \mathbf{r}}
\end{gathered}
$$

Substituting $\ddot{\mathbf{r}}$ from equation (66) into equation (50) gives the equation to be solved for $\lambda$ in the predictor. The transition from slip to stick can be determined by comparing the tip- and rotor velocities and the transition from stick to slip can be determined by comparing $\mathbf{F}_{\mu, \text { stick }}$ with the normal force.

\section{CONCLUSION}

Using Hamilton's principle and the Rayleigh-Ritz method the resonator of the piezomotor was modelled and the Lagrange Multiplier method was used to model the contact behaviour. It was shown that the ratio of resonance frequencies and the shape of the tip trajectory can be optimised by changing the position of piezoelements. Further improvements to obtain a factor two ratio of the resonance frequencies are possible. The Lagrange Multiplier method results in some none-physical contact forces which suggests that the contact model should include the contact stiffness. This issue will investigated in the future. Simulations show that stick occurs therefore a method for including stick in the model was suggested.

\section{REFERENCES}

Andersen, Brian, Mogens Blanke, Jan Helbo, Arkadii Borisovich Smirnov, Anatoly Aleksandrovi Yerofeyev and Sergey Anatoljevich Yerofeyev (2000). Vibration Actuator. PCT, patent application, patent nr. WO0030186 A.

Campbell, Stephen L. and B. Leimkuhler (1991). Differentiation of Constraints in Differential-Algebraic Equations. Mechanics of Structures and Machines 19(1), 19-39.

Fung, Rong-Fong, Chih-Min Yao and Dong-Guey Chang (1999). Dynamic and Contact Analysis of a Bimodal Ultrasonic Motor. IEEE Transactions on Ultrasonics, Ferroelectrics and Frequency Control 46(1), 47-60.

Gear, C. W., B. Leimkuhler and G.K. Gupta (1985). Automatic Integration of Euler-Lagrange equations with constraints. Journal of Computational and Applied Mathematics (12/13), 77-90. Hagood, Nesbitt W., Walter H. Chung and Andreas von Flotow (1990). Modelling of Piezoelectric Actuator Dynamics for Active Structural Control. Journal of Intelligent Materials, Systems and Structures 1, 327-355.

Hatman, V. G., I. Haque and A. Bagchi (1996). Dynamics of a Flexible Rotating Beam Interacting with a Flat Rigid Surface, Part II: Numerical Solution. Journal of Sound and Vibration 194(5), 671-683.

Wallaschek, Jörg (1998). Contact mechanics of piezoelectric ultrasonic motors. Smart Materials and Structures 7(3), 369-381. Yen, Jeng, Edward J. Haug and T. O. Tak (1991). Numerical Methods for Constrained Equations of Motion in Mechanical System Dynamics. Mechanics of Structures and Machines 19(1), 41-76.

Zharii, Oleg Yu. (1996). An exact solution of a time-dependent frictional contact problem for two elastic spheres. International Journal of Engineering Science 34(5), 537-548. 